Review Article

\title{
Hematological Malignancies: Epidemiological, Clinical, Biological, Therapeutic and Evolutionary Aspects
}

\author{
Mouhib Hanane", Zahir Hanane, Mouayche Ikhlas, Yahyaoui Hicham, Ait Ameur Mustapha, \\ Chakour Mohammed
}

Laboratory of Hematology, Avicenna Military Hospital, Mohammed VI UHC, Marrakech, Morocco

Email address:

mouhib.hanane1@gmail.com (M. Hanane)

${ }^{*}$ Corresponding author

\section{To cite this article:}

Mouhib Hanane, Zahir Hanane, Mouayche Ikhlas, Yahyaoui Hicham, Ait Ameur Mustapha, Chakour Mohammed. Hematological Malignancies: Epidemiological, Clinical, Biological, Therapeutic and Evolutionary Aspects. American Journal of Laboratory Medicine. Vol. 4, No. 1, 2019, pp. 19-23. doi: 10.11648/j.ajlm.20190401.13

Received: February 10, 2019; Accepted: March 12, 2019; Published: April 13, 2019

\begin{abstract}
Hematological malignancies are diseases, which include all cancers of blood and lymphoid organs. They result from a proliferation of mature blood cells or immature blood cells. However, in all cases, these blood cells escape the normal regulation. This work aims to determine the types and frequencies of diagnosed malignancies. It's supported by the clinical hematological department of the Military Hospital Avicenna in Marrakech. We conducted a retrospective study over a period of 4 years from January, 1st 2012 to December, 31st 2015. We used an operating record, which includes epidemiological, clinical, biological, therapeutic and evolutionary criteria. During this period, we found that 70 cases were diagnosed and treated in the clinical hematological department of the HMA. We recorded 26 cases of multiple myeloma (37\%), 23 cases of leukemia (33\%), 19 cases of lymphoma (including 15 cases of non-Hodgkin lymphoma (21.4\%) and 4 cases of Hodgkin lymphoma $5.7 \%$ ), 1 case of myelodysplastic syndrome (1.4\%) and 1 case of polycythemia vera (1.4\%). This work allowed us to note a significant increase in malignancies in this study. Hence the importance of raising the awareness of health professionals and raising awareness on a larger scale of the general population to improve the time of care and indirectly improve the prognosis of these diseases.
\end{abstract}

Keywords: Hematological Malignancies, Epidemiology, Biology

\section{Introduction}

Hematological malignancies include all cancers of the blood and lymphoid organs. They result from a proliferation of mature blood cells (responsible for chronic haemopathies of slow evolution) or immature (causing acute haemopathies of rapid evolution) but which, in any case, escape the normal regulation. [1]

Most hematological malignancies result most often from mutations that occur in a cell clone, following damage to the DNA during its duplication during mitosis. This is usually a series of successive mutations that seem to confer full malignity to the clone concerned. [2]

International classifications of hematological malignancies are becoming more complex. This is explained by the better knowledge of normal and pathological hematopoiesis thanks to the development of molecular biology techniques, making it easy to study the genome and different cell signaling pathways. [3]

The hematological malignancies are serious diseases with often pejorative prognosis. In recent decades, a better understanding of the physiopathological mechanisms has led to the development of new treatments, which have upset the management of some previously incurable haematopathies (eg tyrosine kinase inhibitors in chronic myeloid leukemia, ATRA in AML3). [1]

The objective of our study is to report the epidemiological, clinical, hematological, therapeutic and evolutionary aspects of the hematological malignancies observed at the Avicenne Military Hospital in Marrakech. 


\section{Patients and Methods}

Our work is a retrospective study of patients in care in the clinical hematology department of the Avicenne Military Hospital in Marrakech, during the period from January 1, 2011 to December 31, 2015.

Excluded from our study, cases of hematological malignancies diagnosed at the HMA and evacuated at the University Hospital of Marrakech or Mohammed V Hospital of Rabat.

The collection of data for each patient was done on the basis of medical records.

The information collected was recorded on a farm return that included epidemiological, clinical, biological, therapeutic and evolutionary criteria.

The data entry and analysis was done using the "Microsoft Office Excel 2010" software for Windows version 10.0.

\section{Results}

Over the period of the study, we collected 70 cases. The average age of patients is 51 years with extremes ranging from 19 to 83 years and with a predominance of cases whose age range is between 51 to 60 years (Figure 1). 43 patients were male $(61 \%)$ against 27 female $(39 \%)$ with a sex ratio $\mathrm{M} / \mathrm{F}$ of 1.5. Fifty-three percent of our patients were military or ex-military.

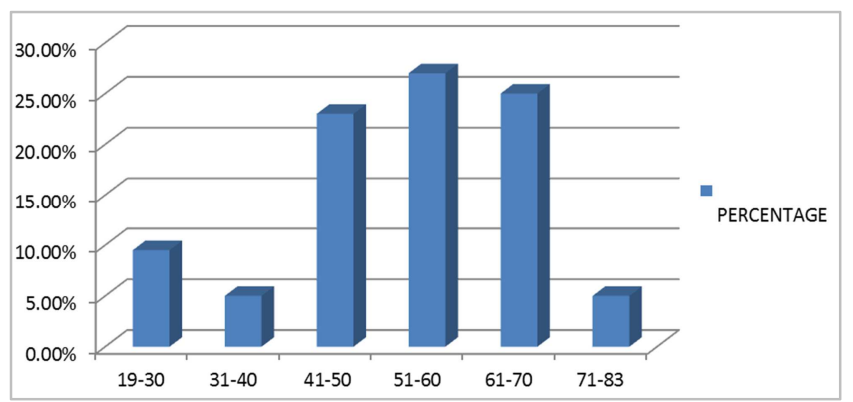

Figure 1. Distribution of haematological malignancies as a function of age.

About half of the patients live in the Marrakech-Safi region (47\%); The city of Marrakech comes in first place with 20 cases, followed by El kelaa with 5 cases, Safi with 3 cases, Aitourir with 2 cases, Bengrir with 1 case, Laattaouia with 1 case and the city of Essaouira with 1 case.

Twenty Six patients, or (37.1\%), have no personal or family history.

The average consultation time was 6 months with extremes ranging from 7 days to 4 years. In $82.8 \%$ of our patients, malignant hemopathy was discovered following functional symptoms; the alteration of the general state comes first (Table 1).

Table 1. Distribution of patients with hematological malignancies according to the reason for consultation.

\begin{tabular}{|c|c|c|c|}
\hline & & number & percentage \\
\hline \multirow{9}{*}{$\begin{array}{l}\text { functional signs discovered in } \\
\text { consultation }\end{array}$} & alteration of the general state & 24 cases & $41,4 \%$ \\
\hline & lymphadenopathy & 16 & $27,6 \%$ \\
\hline & bone pain & 14 & $24,1 \%$ \\
\hline & infectious syndrome & 8 & $13,8 \%$ \\
\hline & hemorrhagic syndrome & 6 & $10,3 \%$ \\
\hline & anemic syndrome & 5 & $8,6 \%$ \\
\hline & pathological fractures & 5 & $8,6 \%$ \\
\hline & abdominal mass & 4 & $6,9 \%$ \\
\hline & other & 3 & $5,2 \%$ \\
\hline \multirow{3}{*}{ lucky find } & Complete blood count (CBC) & 6 cases & $50 \%$ \\
\hline & electrophoresis of proteins & 5 cases & $41,6 \%$ \\
\hline & scanner & 1 case & $8,3 \%$ \\
\hline
\end{tabular}

In this study, multiple myeloma ranked first with 26 cases (37.1\%), followed by leukemias (acute and chronic) with 23 cases $(33 \%)$, followed by lymphoma with 19 cases $(27.1 \%)$. 15 cases of non-Hodgkin's lymphoma (21.4\%) and 4 cases of
Hodgkin's lymphoma (5.7\%). Myelodysplastic syndromes come last with only one case $(1.4 \%)$ and only one case of polycythemia vera. (Figure 2 )

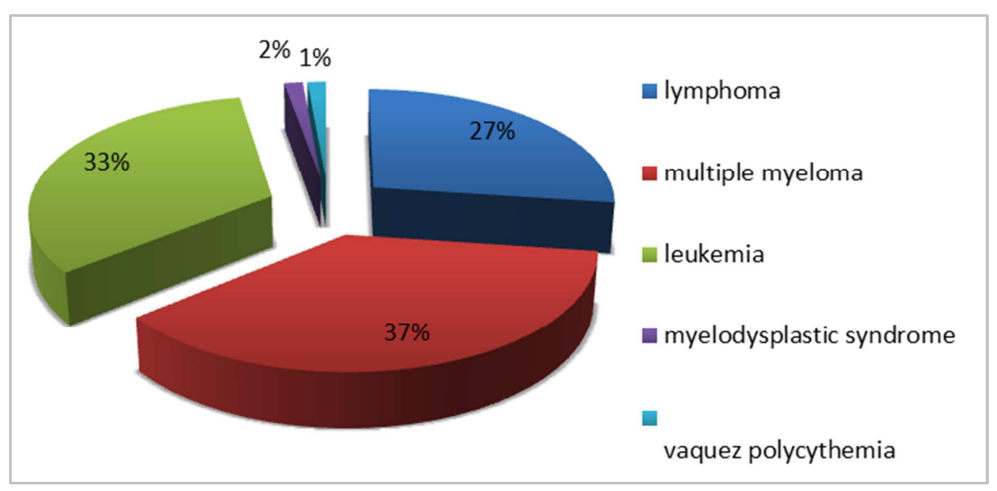

Figure 2. Distribution of cases of hematological malignancies by histological type. 
On the hematological level, the myelogram and the osteomedullary biopsy made it possible to posit the positive diagnosis in respectively $61 \%$ and $39 \%$ of the cases.

The impact assessment revealed anemia in $64.3 \%$ of cases, leukopenia in $18.5 \%$ of cases and thrombocytopenia in $33 \%$ of cases.

The inflammatory balance including sedimentation rate (SV) and C-reactive protein (CRP) were high in $64 \%$ and $60 \%$ of cases, respectively. Renal failure was found in $17 \%$ of cases, $7 \%$ of our patients had hypercalcemia and $11.5 \%$ had longer coagulation times.

Therapeutically, $97 \%$ of patients received chemotherapy, $47 \%$ received radiotherapy, $13 \%$ hematopoietic stem cell transplantation, and $7 \%$ of patients received simple monitoring (Watch and wait).
The evolution was marked by complete remission in 34 patients, ie $48 \%$, partial remission in 15 patients $(21 \%)$, relapse in 3 patients $(4 \%)$, stability in 2 patients $(3 \%)$ and death in 10 patients $(14 \%)$.

\subsection{Multiple Myeloma (MM)}

For 4 years, the clinical hematology department collected 26 cases of multiple myeloma, with a male predominance. The sex ratio $\mathrm{M} / \mathrm{F}$ is 1.36 . The average age was 59 years old with extremes ranging from 42 to 83 years old.

The number of multiple myeloma cases has increased significantly, from a single case in 2012 to 13 cases in 2015 (Figure 3). The study of the seasonal distribution of multiple myeloma cases revealed a peak in April. (Figure 4).

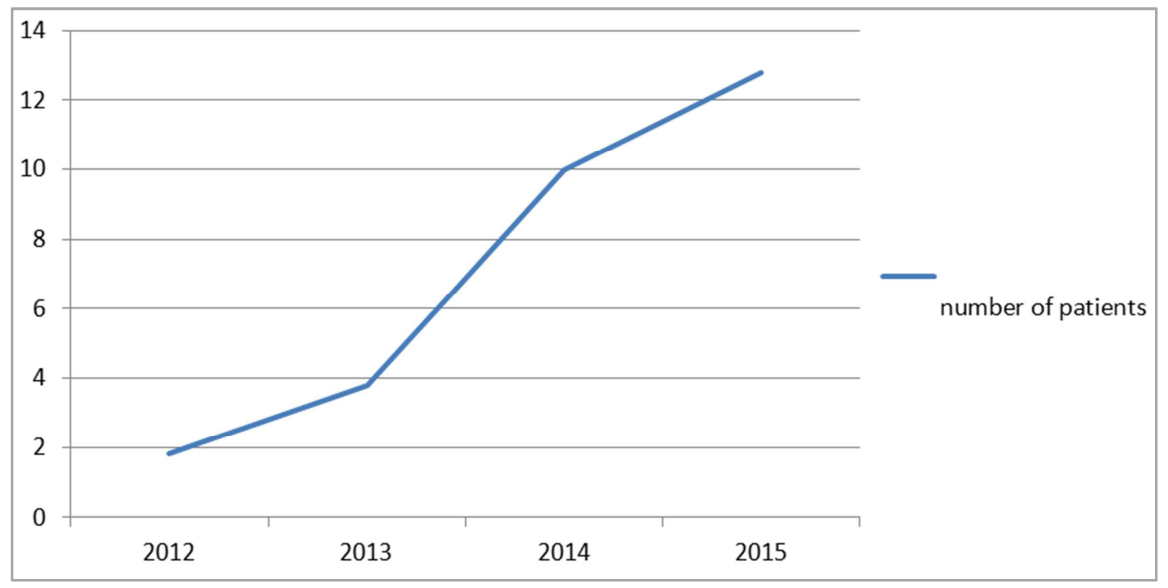

Figure 3. Frequency of Multiple Myeloma by Years.

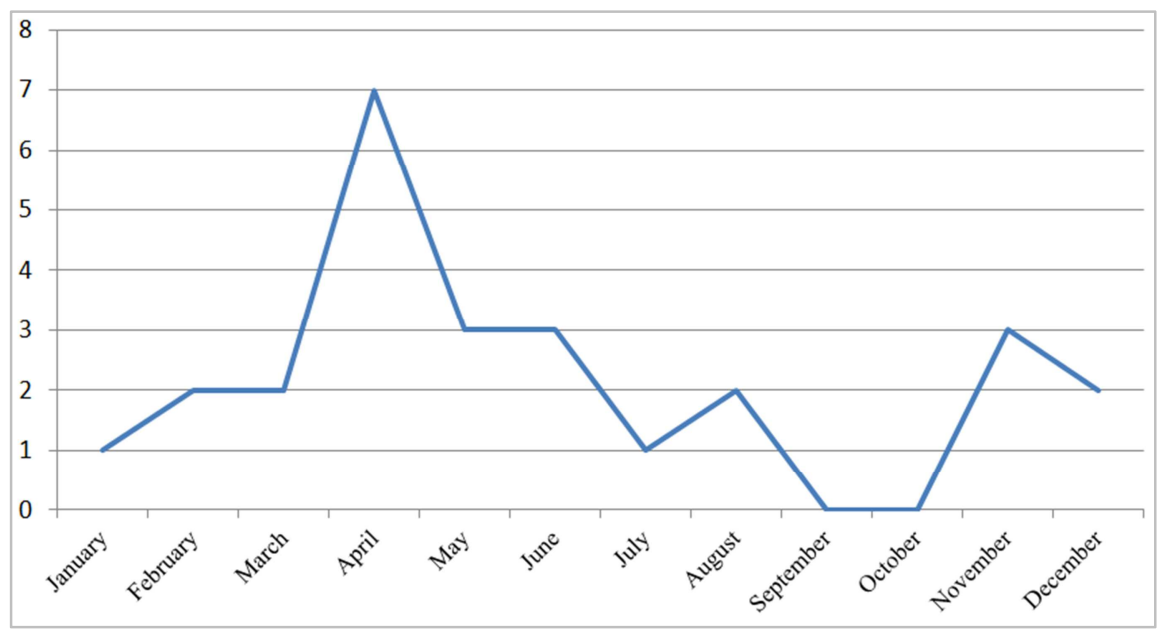

Figure 4. Frequency of multiple myeloma in terms of months.

$73.1 \%$ of the cases of recorded myeloma were symptomatic myeloma (Table 2). Therapeutically, 24 patients received chemotherapy, five of whom received a stem cell transplant and 2 patients were treated with radiation therapy for analgesic purposes. Remission was observed in 19 patients (73\%) of whom 10 partial (38.4\%) and 9 complete $(34.6 \%) .4$ patients are being followed and the death of $3(11.5 \%)$ is deplored.
Table 2. Distribution of myeloma according to the IMWG classification.

\begin{tabular}{lll}
\hline & number of patients & percentage \\
\hline $\begin{array}{l}\text { MGUS } \\
\text { monoclonal gammopathy of }\end{array}$ & 2 & 7.7 \\
$\begin{array}{l}\text { undetermined significance } \\
\text { asymptomatic myeloma }\end{array}$ & 5 & 19.2 \\
symptomatic myeloma & 19 & 73.1 \\
\hline
\end{tabular}




\subsection{Acute Myeloid Leukemia (AML)}

We noted 12 cases of acute leukemia, acute myeloblastic leukemia is the most common type of leukemia in our series accounting for $83.3 \%(\mathrm{n}=10)$ of acute leukemias. The average age of the patients was 50 years with extreme ages of 19 to 71 years. There was a male predominance with a sex ratio of 1.5. Cytologically acute myelogenous leukemia type 2 (AML2) is the most common (30\%). All patients received chemotherapy with complete remission in 4 patients, partial remission in 1 patient, relapse in 2 and death of 3 patients.

\subsection{Acute Lymphoblastic Leukemia (ALL)}

We found 2 cases of ALL aged 19 and 51 respectively. These 2 patients received chemotherapy and one of them also received prophylactic radiotherapy of the central nervous system. The evolution was marked by complete remission in both patients.

\subsection{Chronic Myeloid Leukemia (CML)}

Chronic myeloid leukemia (CML) is the most dominant myeloproliferative disorder in our series. We collected 5 cases of CML or $7 \%$ of all hematological malignancies. Patients had an average age of 55 with extremes ranging from 42 to 65 years old. A predominance of the male sex was noted, representing $80 \%$ of patients with CML.

\subsection{Lymphomas}

During our study period, we recorded 19 cases of lymphoma or $27.1 \%$, including 15 cases of non-Hodgkin's lymphoma (NHL) (21.4\%) and 4 cases of Hodgkin's lymphoma (5.7\%). Almost half of the cases are diagnosed in Stage IV according to Costwolds' Ann Arbor classification. Histologically, $86.6 \%$ of NHL were B-cell lymphomas. Ganglionic localization represents $66.7 \%$ of NHLs, while extra-ganglionic sites represent $33.3 \%$ of the total number of NHL cases with predominance of digestive localizations. The number of NHL cases went from a single case in 2012 to 6 cases in 2015. All patients received chemotherapy. Three benefited from rituximab in addition to chemotherapy. The evolution was marked by the complete remission of $86.6 \%$ of cases $(n=13)$ and the death of 2 patients was $13.4 \%$.

\subsection{Myelodysplastic Syndrome}

During the period of our study, a case of myelodysplasia was found; in a man aged 29 years. The patient received chemotherapy and a stem cell transplant and the evolution was marked by the stability of the disease.

\section{Discussion}

The hematological malignancies are malignant proliferations of cells belonging to the different cell lines constituting the hematopoietic tissue. Leukemias, lymphomas and multiple myeloma are distinguished. [4]

In our study, as well as that of the University Hospital of
Marrakech and Casablanca, the majority of patients with hematological malignancies were under 65 years of age $[5,6]$. In Lower Normandy, this distribution is reversed; the majority of cases are older than 64 years [7]. This is likely because the Moroccan population is younger than the French population. In our series, hematological malignancies are more common in males, as well as in all literary series. $[1,7]$

Multiple myeloma is a malignant plasmocytic proliferation of the bone marrow characterized by the production of complete or incomplete monoclonal immunoglobulin (Ig) and by the high frequency of lytic bone lesions. [8]

It can be diagnosed de novo or in the course of the evolution of previously known monoclonal gammopathy. Multiple myeloma accounts for about $1 \%$ of all cancers and $10 \%$ of all hematological malignancies, making it the most common hematological malignancy after lymphoma [9]. Indeed, in our series, multiple myeloma represents the most common malignant haematological disease. Contrary to this, the studies carried out at the national level in Casablanca and Marrakech and at the international level in Lower Normandy and Bamako, show that it is ranked in the $3 \mathrm{rd}$ rank $(37 \%$ of the hematological malignancies) [5-7]. The incidence of multiple myeloma in Morocco is not yet known given the absence of a national registry of tumor diseases. However, there is a Rabat cancer register showing that the incidence of multiple myeloma in 2005 was 2.12 new cases per 100,000 population for men, and $0.94 / 100,000$ for women [11]. In our study, the number of multiple myeloma cases increased significantly from one case in 2012 to 13 cases in 2015 .

The average age of patients with multiple myeloma (MM) is 65 at the time of diagnosis [10]. The incidence of this disease increases with age: 7 per 100,000 at age 50,20 per 100,000 at age 80. MGUSs (Monoclonal Gammopathies of Unknown Significance) are common and their frequency also increases with age: $1 \%$ to 50 years, $5 \%$ to 80 years [10]. In our series the average age of myeloma patients was 59 years old.

Acute leukemia (LA) is a heterogeneous group of malignant haematological disorders characterized by clonal and uncontrolled proliferation of hematopoietic precursors blocked in their differentiation. [12]

There are two main types of acute leukemia according to the myeloid or lymphoid nature of blast proliferation:

(1) Acute lymphoblastic leukemia (ALL).

(2) Acute myeloblastic leukemia (AML).

AML are more frequent than all LALs $(70 \%$ of LA cases are myeloid) and affect mainly adults. They represent 75 to $80 \%$ of LA cases in adults and 15 to $20 \%$ in children. [12]

In our series AML is the most common type accounting for $83.3 \%$ of acute leukemias. In Lower Normandy, we find similar results: $72 \%$ of LA are LAM. [7]

ALL are more common in children than in adults: about $75 \%$ of reported cases occur in patients younger than 18, with a peak frequency of 2 to 5 years [12], hence the results. of our series or only 2 cases were found, ie $3 \%$ of the hematological malignancies.

Lymphomas are neoplasms that develop from lymphoid cells at different stages of their maturation giving rise to $\mathrm{B}$ or $\mathrm{T}$ lines. 
Two large groups are individualized: non-Hodgkin's lymphoma (NHL) and Hodgkin's lymphoma. [13] In 2002, the age-adjusted incidence of non-Hodgkin's lymphoma was about 23.2 per 100,000 for men and 16.3 per 100,000 for women. The incidence increases considerably with age; it is higher among whites than among other ethnic groups [14]. According to the cancer registry of Greater Casablanca in 2012, the incidence of NHL has increased significantly from 5.4 in 2005 to 7.7 in 2007 in men and 3.7 in 2005 to 5.1 in women [6]. In our series, NHL is the most represented lymphoma with 19 cases or $27 \%$.

\section{Conclusion}

Through the results of our small series and those of the similar work of the hospitals of Marrakech and Casablanca, it seems that the incidence of hematological malignancies has significantly increased in recent years, and affects a younger population compared to European studies.

This encourages the development of appropriate care strategies and the development of national registries that can provide clearer insights into the epidemiological data of different blood diseases (incidence and prevalence), and assist in the development of prevention and control protocols screening.

\section{References}

[1] Mahboub. F. Z, Elkhattabi. W, L'youssfi. H, Aichaneet. A, Afif. H. Diagnostic positif et évolution des hémopathies malignes (à propos de 132 cas). Rev Mal Respir 2015; 32: A130-A130.

[2] Delabesse E. Marqueurs génétiques des hémopathies. EMCBiologiemédicale 2018; 13: 1-8.
[3] Thachil. J, Bates. I. Approach to the Diagnosis and Classification oh Blood Cell Disorders. Practical Haematology 2017; 23: 497-510.

[4] Questel. F. Hémopathies malignes d'origine professionnelle. Pathologie professionnelle et de l'environnement 2011; 6: 1-12.

[5] RHAFEL. A. Bilan d'activité du service d'hématologie du CHU Mohammed VI (2009-2013). Thèse de doctorat en médecine, sous la direction du Pr. L. Mahmal, Marrakech, Faculté de médecine et de pharmacie, 2014; 223: 42-43.

[6] Harif. M, Benider. A, BennaniOthmani. M. et al. Registre des cancers de la région du Grand Casablanca 2012; 88: 72-78.

[7] Troussard. X, Duchenet. V, Cornet. E, Mouchel. D, Malet. M, Collignon. A. Epidémiologie des hémopathies malignes en Basse-Normandie. Rev. épidémiol. santé publique 2009; 57: 151-158.

[8] Fouquet. G, Guidez. S, Richez. V, Systchenko. T, Gruchet. C, Moya. N et all. Myélome multiple. EMC- Hématologie 2017; 12: 1-26.

[9] Arnulf. B. Myélome multiple des os. Rev Prat 2013; 63: 1018.

[10] Laroche. M. Myélome multiple des os (maladie de Kahler). Rhumatologie pour le praticien 2018; 21: 257-263.

[11] Tazi. M, Benjaafar. N, er-raki. A. Incidences des cancers à Rabat 2005; 84: 82-82.

[12] Duployez. N, Decool. G, Preudhomme. C. Classification et facteurs pronostiques des leucémies aiguës. EMCHématologie 2018; 13: 1-14.

[13] Opsomer. M.-A, Fagnoni. P, Bastie Pr. J.-N. Lymphomes. Pharmacie clinique pratique en oncologie 2016; 26: 211-211. 